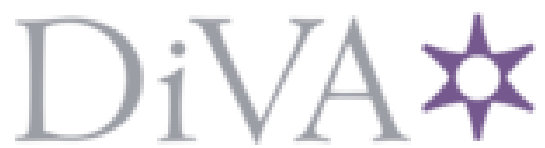

http://www.diva-portal.org

This is the published version of a paper presented at NEW2AN 2O18, ruSMART 2018 .

Citation for the original published paper:

Kuzminykh, I., Carlsson, A., Franksson, R., Liljegren, A. (2018)

Measuring a LoRa Network: Performance, Possibilities and Limitations.

In: Galinina O., Andreev S., Balandin S., Koucheryavy Y. (ed.), Internet of Things,

Smart Spaces, and Next Generation Networks and Systems. NEW2AN 2O18,

ruSMART 2018. Lecture Notes in Computer Science, vol 11118. (pp. 116-128). Cham

Lecture Notes in Computer Science

https://doi.org/10.1007/978-3-030-01168-0_11

N.B. When citing this work, cite the original published paper.

Permanent link to this version:

http://urn.kb.se/resolve?urn=urn:nbn:se:bth-17129 


\title{
Measuring a LoRa Network: Performance, Possibilities and Limitations
}

\author{
Anders Carlsson ${ }^{10000-0002-9328-9143]}$, Ievgeniia \\ Kuzminykh $^{1[0000-0001-6917-4234]}$, Robin Franksson ${ }^{1}$, and Alexander Liljegren ${ }^{1}$ \\ Blekinge Institute of Technology, Karlskrona, Sweden Campus Grasvik, SE- 37141 \\ anders.carlsson@bth.se
}

\begin{abstract}
Low power wide area (LPWA) technologies becomes popular for IoT use cases because LPWA is enable the broad range communications and allows to transmit small amounts of information in a long distance. Among LPWA technologies there are LTE-M, SigFox, LoRa, Symphony Link, Ingenu RPMA, Weightless, and NB-IoT. Currently all these technologies suffer from lack of documentation about deployment recommendation, have non-investigated limitations that can affect implementations and products using such technologies. This paper is focused on the testing of LPWAN LoRa technology to learn how a LoRa network gets affected by different environmental attributes such as distance, height and surrounding area by measuring the signal strength, signal to noise ratio and any resulting packet loss. The series of experiments for various use cases are conducted using a fully deployed LoRa network made up of a gateway and sensor available through the public network. The results will show the LoRa network limitation for such use cases as forest, city, open space. These results allow to give the recommendation for companies during early analysis and design stages of network life circle, and help to choose properly technology for deployment an IoT application.
\end{abstract}

Keywords: IoT · LoRa · LPWAN · Transmission Range · Arduino . Raspberry $\mathrm{Pi}$

\section{Introduction}

The interest and applications for Internet of Things has in recent years increased significantly. According to the forecast about IoT market from the global leaders such as Juniper Research, Research Nester, Cisco, Ericsson, Gartner, in just a couple of years tens of billions of different kind of things are estimated to be connected $[1,2]$. Most of these devices will be deployed in WAN (Wide Area Network) solutions [3]. This introduce a demand for new communication standards that targets the key features needed to deploy the technology in society such as low power consumption and long range coverage. This led to the creation of LPWANs (Low Power Wide Area Network) which is designed for low bit rate long range communication [4]. The goal is to replace devices that today rely on 
cellular communications like GSM and $4 \mathrm{G}$ at a higher cost and power consumption with new ones that adapt this low bit rate communication style to provide battery lifetimes up towards 10 years while maintaining a communication range up to $30 \mathrm{~km}$ under optimal circumstances.

Due to the LPWA technology being new and not thoroughly fleshed out together with being accessible and completely open to the public this paper will have a focus on measuring and evaluating one of these LPWAN technologies LoRa and its communication protocol LoRaWAN. Currently, the research on LoRa is very limited and mainly focused on how certain factors like temperature, humidity and precipitation affect the maximum range and battery life while the research on how a LoRa network actually performs and the research in this field is limited mostly by simulations and theories.

In this work we provide further insight over how performance of a LoRa network is affected by placement by showing signal quality, signal-to-noise ratios and packet loss in various environmental areas. By analyzing performance on the end-devices and gateways we hope to bring some light over how such kinds of systems should be set up to fit different use cases and also giving a hint to whether the technology is feasible given a specific use case.

\section{Wireless technologies for IoT}

\subsection{LPWAN technologies}

Currently, in the world a great variation of technologies is used for the IoT where each is made to fit a certain domain. For smart homes it fits Wi-Fi when possible and Bluetooth when not. A relatively new candidate, however, used for several home products is ZigBee which low-power and low-rang, it perfect fits for applications like home automation i.e. lighting, temperature, security and sensors. As distance increases the possibilities of using such technologies gets limited since both Wi-Fi and ZigBee are limited up to $100 \mathrm{~m}$, so for products that require a higher range the cellular networks like GSM up to 5G are used, these however come at a heavy price in both licensing costs and battery lifetime [5]. The interest in adding this missing piece in radio communication technologies led to the creation of LPWANs. A comparison of the trade-offs in range and data rate for the various technologies can be seen on Fig. 1.

LPWAN or Low-Power Wide-Area Network is an umbrella term for technologies that focus on having very high power efficiency while maintaining a high transmission range to meet this goal a sacrifice in data rate is made. The goal of these technologies is to fill the niche of products that require long battery lifetime, have low duty cycles and require medium or long range [6]. Examples of such products can be agriculture and industrial sensors, smart cities applications such as traffic, trashcan and parking sensors. There are several variants of these LPWAN technologies such as LTE-M, SigFox, LoRa, Symphony Link, Ingenu RPMA, Weightless $\mathrm{W}, \mathrm{N}$ and $\mathrm{P}$, and NB-IoT.

Common features of LPWAN technologies are summarized in Table 1. 


\begin{tabular}{|c|c|c|c|c|c|c|c|c|}
\hline 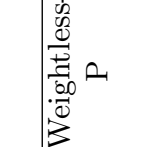 & 袋 & 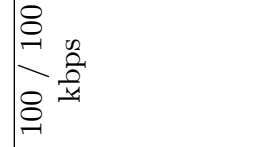 & 票 & 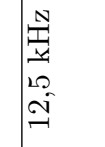 & 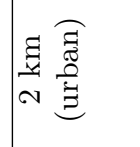 & 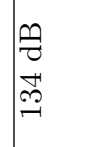 & 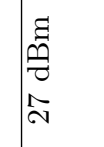 & \\
\hline 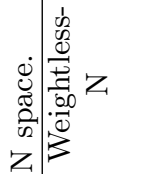 & $\begin{array}{l}\frac{1}{n} \\
\hat{D}^{2} \\
\hat{0}\end{array}$ & 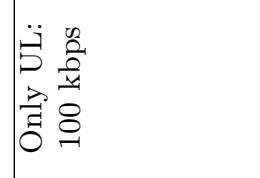 & 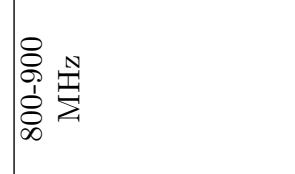 & 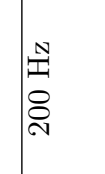 & 亲 镸 & 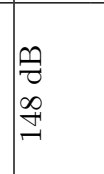 & 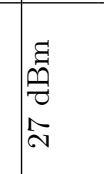 & 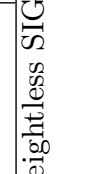 \\
\hline 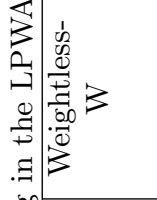 & 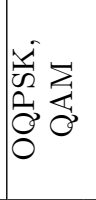 & 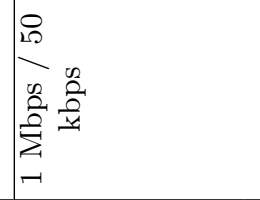 & 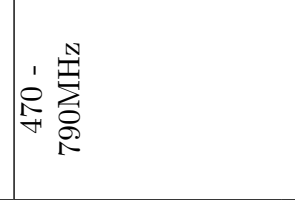 & $\mid \begin{array}{l}N \\
\sum_{1} \\
\infty \\
0 \\
0 \\
0\end{array}$ & 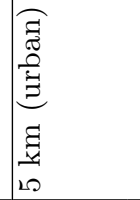 & 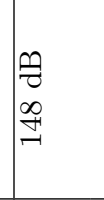 & 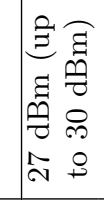 & \\
\hline 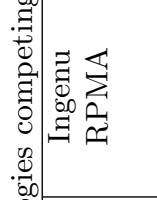 & 車 & 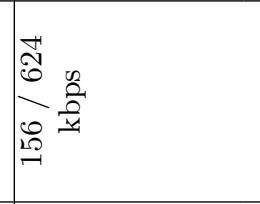 & 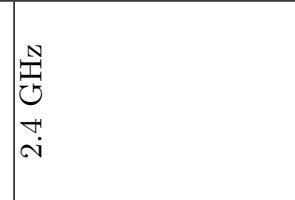 & $\sum_{-1}^{N}$ & 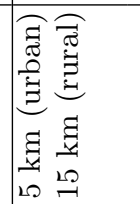 & 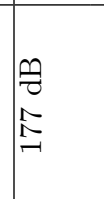 & 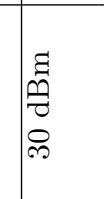 & 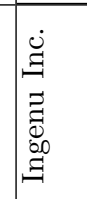 \\
\hline 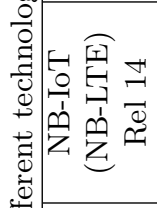 & 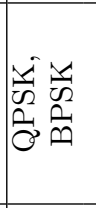 & 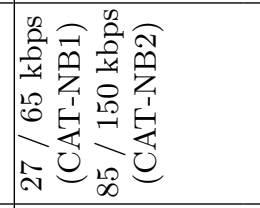 & 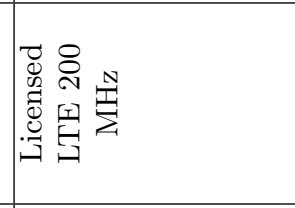 & 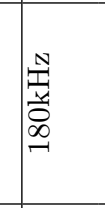 & 嘅 & 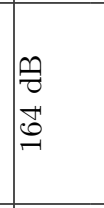 & 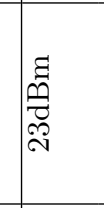 & 总 \\
\hline 焉 & 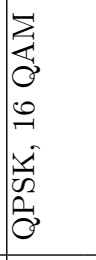 & 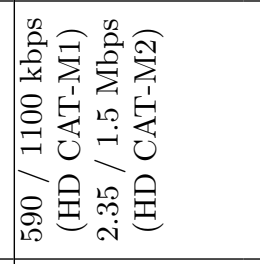 & 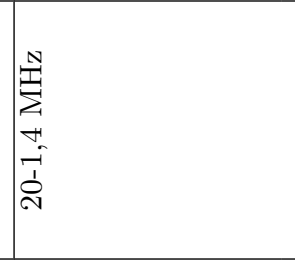 & $\sum_{10}^{N}$ & $\frac{g}{9}$ & 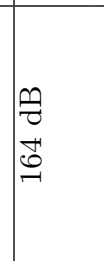 & 泀 & 总 \\
\hline 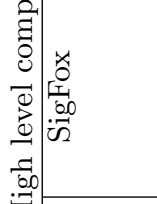 & 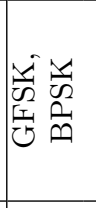 & 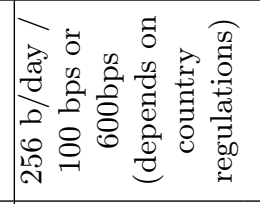 & 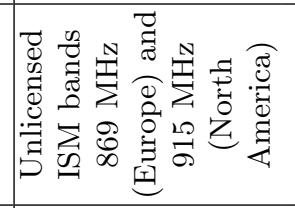 & 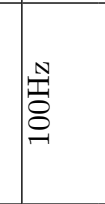 & 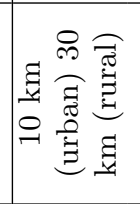 & 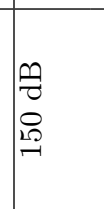 & 泀 & 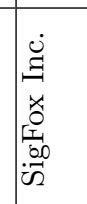 \\
\hline 党 & $\begin{array}{l}\mathscr{W} \\
z^{2}\end{array}$ & 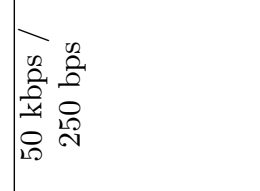 & 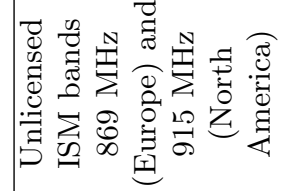 & 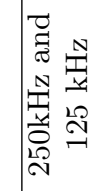 & 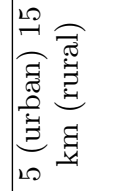 & 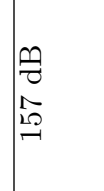 & 泀 & 造 \\
\hline 总 & 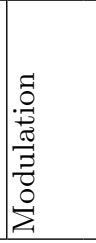 & 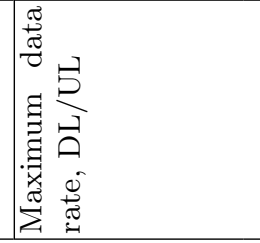 & 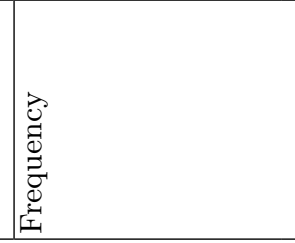 & 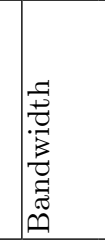 & 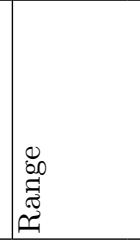 & 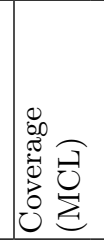 & 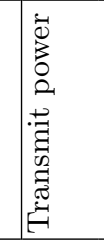 & 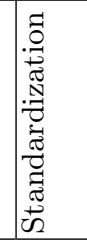 \\
\hline
\end{tabular}




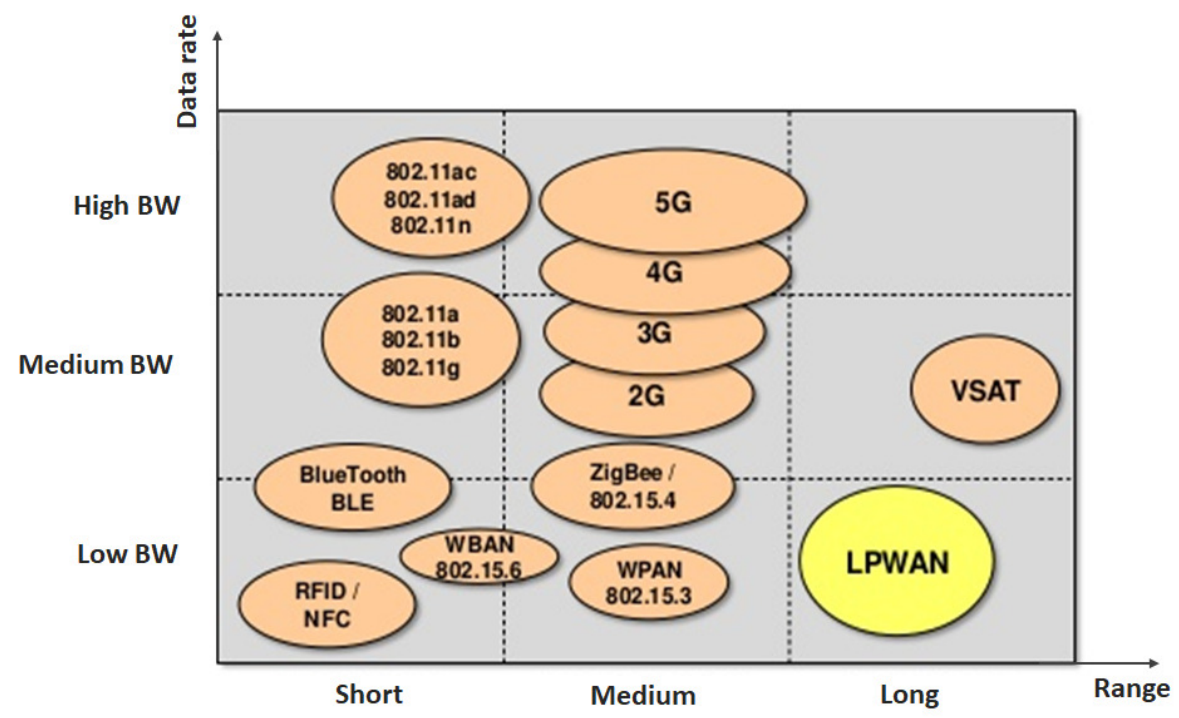

Fig. 1. Data rate vs. range capacity of radio communication technologies.

Lora is a technology based on open protocol but limited to Semtechs chips. It has low data rate that varies according to the $\mathrm{SF}$ and the bandwidth (27 kbps with $\mathrm{SF}=12$ and $500 \mathrm{kHz}$ channel or $50 \mathrm{kbps}$ with FSK). The LoRa uses Chirp Spread Spectrum (CSS) modulation when the signal is modulated by chirp pulses to increase resilience against interference, Doppler effect and multipath.

SigFox is not an open-protocol but one of the most developed. It is not limited to particular chips and the vendors can produce chips with SigFox compatible radios. SigFox is not cheap technology, it sells its network as a service. It is extremely narrow band and provide up to $30 \mathrm{~km}$ range. It has daily messages limit that depends on the contract you subscribed for. The maximum limit is then 140 messages in UL with a payload up to 12 bytes and 4 messages in DL with a payload of 8 bytes. These restrictions and business model shifted the interest to LoRaWAN that is considered more flexible and open.

LTE-M and NB-LTE (NB-IoT) from 3GPP group are part of LTE infrastructure. They use licensed spectrum and an LTE-based synchronous protocol and provide optimal QoS at the expense of cost.

Ingenu technology is open standard based on Random Phase Multiple Access (RPMA). It has high energy consumption thats why is not so popular.

The Weightless standards has three types of protocols: Weightless-W, Weightless$\mathrm{N}$ and Weightless-P. Weightless- $\mathrm{W}$ and Weightless-P have bidirectional communication. Weightless- $\mathrm{N}$ was developed to increase the transmission range of Weightless-W, decrease data rate and reduce the power consumption. Weightless- 
N operates in Ultra Narrow Band (UNB) 800-900 MHz and provides only uplink communication.

\subsection{LoRa and LoRaWAN}

LoRa is a long-range wireless communications technology promoted by the LoRa Alliance. It operates on the license free ISM bands and uses Chirp Spread Spectrum (CSS) radio modulation technique on physical layer, and a MAC layer protocol LoRaWAN. Depending on the frequency band the LoRaWAN duty cycle can be $0.1 \%, 1 \%$ or $10 \%$ but recommended is less than $1 \%$. Value of duty cycle is considered as delay between the successive frames sent by the end node. If the value is $1 \%$ the device will have to wait 100 times of the duration of the last frame before sending again in the same channel. Last document [7] from LoRa Alliance specifies frequency and bandwidth parameters for different regions over the world. Also, document [8] describes all parameters required to the device communication on physical and MAC levels, the formats of messaging, explains how devices of different types should operate.

The payload of each transmission can range from 2 to 255 octets, and the data rate can reach up to $50 \mathrm{Kbps}$ when channel aggregation is used. The maximum data rate varies according to the SF and the bandwidth. The spreading factor is a logarithm, in base 2, of the number of chirps per symbol, i.e. the number of bits encoded into each symbol. And a symbol is an instantaneous change in frequency. So, in an unique variation of frequency it is associated not only one bit, but more (e.g. in SF7 it is 7 bits). The chirp rate depends only on the bandwidth: the chirp rate is proportional to the bandwidth (one chirp per second per Hertz of bandwidth). Changing of spreading factor is a process in which we add more information, redundancy, to obtain a better receive sensitivity. The relationship between the data bit rate, sensitivity and chip rate for LoRa modulation can be observed in Table 2 .

Table 2. Spread factor specification.

\begin{tabular}{|l|c|c|l|}
\hline Mode & Equivalent bit rate (kbps) & Sensitivity $(\mathrm{dBm})$ & $(\mathrm{dB})$ \\
\hline FSK & 1,2 & -122 & - \\
LoRa SF $=12$ & 0,293 & -137 & +15 \\
LoRa SF $=11$ & 0,537 & $-134,5$ & $+12,5$ \\
LoRa SF $=10$ & 0,976 & -132 & +10 \\
LoRa SF $=9$ & 1757 & -129 & +7 \\
LoRa SF $=8$ & 3125 & -126 & +4 \\
LoRa SF $=7$ & 5468 & -123 & +1 \\
LoRa SF $=6$ & 9375 & -118 & -3 \\
\hline
\end{tabular}

On the MAC layer LoRa uses the communication protocol LoRaWAN. So again, using the WiFi analogy, if LoRa is the WiFi connection, LoRaWAN is the IP protocol. It is intended primarily for wireless long battery time devices 
and targets key points of the Internet of Things such as mobility, localization and bi-directional communication. LoRaWAN [8] specifies three different types of end-devices to address the various needs of applications. Class A is bi-directional, Class $\mathrm{B}$ is bi-directional with scheduled receive slots and class $\mathrm{C}$ is bi-directional with maximal receive slots. Last type has the maximum power consumption because device is almost all time in open receiving mode.

LoRaWAN doesnt support device-to-device communications, data can be transmitted only in way device-to-gateway, or vice versa. Device-to-device communication could be set up through gateway communication. The architecture of a LoRaWAN network is typically laid out in a star-of-stars topology where gateways act like bridges relaying messages between the end-point devices and a central network server. Devices in a LoraWAN network are remote objects that can range from anything between a thermometer to a geolocation based tracking system.

\section{Related work}

According to [9] many of LPWAN technologies will have a share on the IoT market in the future as each of the technologies are more fit for different use cases where some focus more on a higher battery lifetime while others on maximum range and throughput. A comparison is made for various possible implementations of LPWAN such as smart farming which heavily favors the LoRa and Sigfox as they do not rely on cellular coverage while applications such as a terminal for retailers sale require low latency due to do not limit the number of transactions, in such cases a Narrow Band implementation more attractive but the power consumption is more.

The research of Centenaro in [10] describes how in 2009 led by the company Sigfox there was a huge interest for a new wireless long range and lower bit-rate technology compared to the commonly used GSM and 3G.

LPWAN technologies can suffer greatly at various environmental variables. This is shown by Cattani in [11] in their studies of how variables such as weather conditions, temperature, humidity affect the performance of an LPWAN solution. For example, increasing the temperature by $10 \mathrm{C}$ will reduce the RSSI by $1 \mathrm{dBm}$. The decrease in signal strength caused by temperature change could in theory render a perfectly good LoRa link unusable.

This aspect is also mentioned by Wennerstrm [12] in their long-term study of meteorological affects where they show that in Uppsala, Sweden, the PRR can fluctuate more than $20 \%$ from day to night where a higher fluctuation is present during the dryer and hotter months June, July and August.

Another variable that can highly affect PRR is oversaturation of the network as there is a large risk for package collision when several devices transmit radio signals in the same time. This is shown by Ferre [13] in his mathematical theory and simulation of approximated packet loss. To avoid this congestion a specification was created with recommendation to limit duty cycles up to $1 \%$ for the 
EU bands that allows the device to transmit a signal for 1 time unit every 100 time units [14].

Petjjrvi in the article [15] evaluates a LoRa network performance when endnode devices are mobile which could be a potential use case. Their results indicate that moving at speeds exceeding $40 \mathrm{~km} / \mathrm{h}$ causes the communication performance to deteriorate while speeds around $25 \mathrm{~km} / \mathrm{h}$ the communication is relatively reliable. They discuss that this behavior can be caused by the doppler effect and that lower SF values could be less affected by it.

As LoRa networks are open for anyone to use and neighboring networks introduce interference. Thiemo Voigh [16] investigates these interferences and how they can be avoided using directional antenna or multiple gateways. First method improves RSSI and at the same time reduces interference on the neighboring gateways. Deploying more gateways are economically irrational.

In the work [17] it was tested LoRa network but for particular gateway equipment of Cisco 910 industrial router. The authors evaluated the maximal throughput that a single device can obtain, network coverage and receiver sensitivity. Their testing environment was limited by suburban area of Paris with dense residential dwellings.

\section{Materials and methods}

To provide testing of the LoRa network performance the preparatory step of literature study was conducted and represented in above section. Found discussions about technology limitations and the reasons behind them were figured out. Furthermore, the company supported us in investigation had their own purpose and were interested mostly on abilities of LoRaWAN in range up to $1 \mathrm{~km}$. We will try to adapt our test based on these limitations and reasons.

\subsection{Case study topology}

The first stage of setting up any network is to ensure the necessary components are configured correctly, this, in turn, will ensure the accuracy of the data collected during the investigation and analysis of the network. But as you will note a LoRa network is not too complicated to setup and run as there are very few components to configure.

The topology of the LoRa network used in this study consists of the end-node device and gateway connected trough the public network to PC with analysing and visualization tool for representing measurements (Fig. 2).

The end-node device and gateway are powered by portable power supplies. The end-node device is configured to send a packet every 10 seconds on the 867.10 - 868.50 MHz frequencies. The gateway receives and decodes the packet and the forwarder software forwards the packet to LORIOT.io for presentation on a PC. 


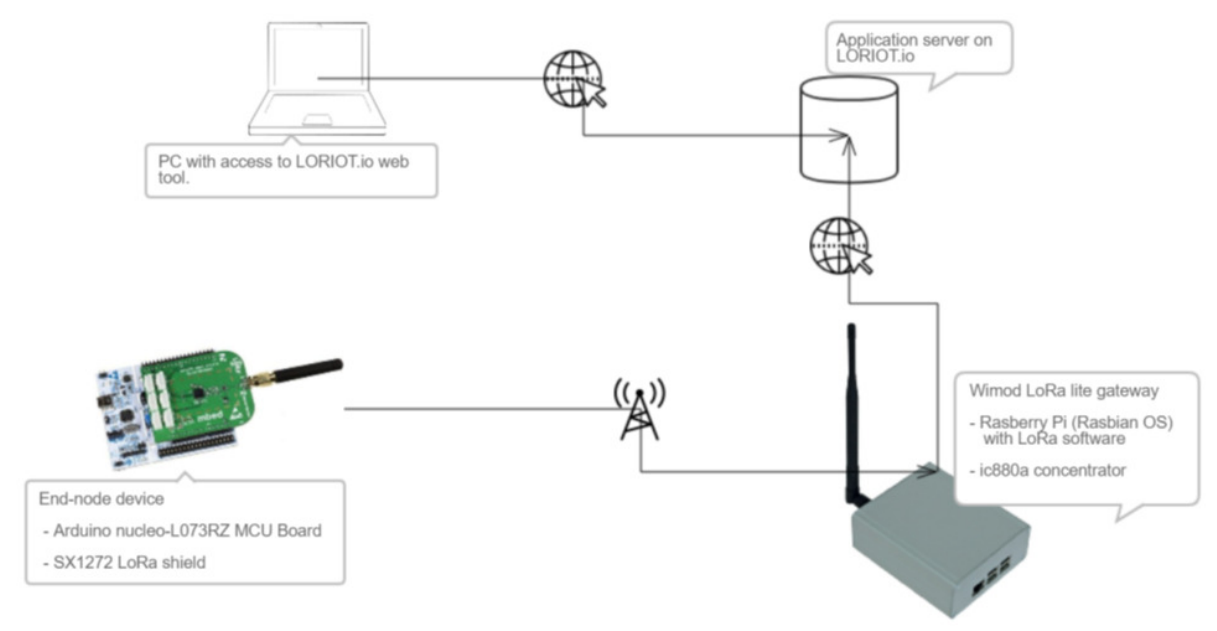

Fig. 2. The topology of the LoRa network.

End-node device In the real use case the end-node device collects data and then send it to the gateway. In our case and for the sake of the experiments this sensor just sends an array of chars.

The LoRaWAN node that we used is set up using the SX1272 LoRa Shield by Semtech and an STMicroelectronics NUCLEO-L073RZ MCU Board. This kit which can be seen in Fig. 2 uses the software I-CUBE-LRWAN provided by ST [18] and the app available called "End-Node". The software has support for SX1276, SX1276 and SX1272 LoRa shields. The IDE Atollic for embedded devices was used to edit the source-code to increase the duty cycle and mocking sensor data, Atollic was also used to flash the code onto the device.

Gateway The gateway that we used is a "Wimod LoRa Lite Gateway" by IMST and is intended for development and evaluation purposes. It consists of a Raspberry Pi and a concentrator iC880A. The LoRa specific source code running on the gateway is provided by the open-source github project LoRa-net [19]. As the gateway is a commercial product it is pre-configured with the appropriate settings upon delivery. The Raspberry $\mathrm{Pi}$ is running Raspbian OS and is preinstalled with the repositories "lora_gateway" and "packet_forwarder" contained in the LoRa-net project.

All experiments were conducted using the following parameters for both enddevice and gateway:

- Bandwidth: 867.1 - 868.5Mhz

- Channel size: $125 \mathrm{kHz}$

- Spreading Factor (SF):variable $=7$ to 12

- Coding Rate:fixed = 4/5

- Transmitting Power: 14dBm 


\subsection{Types of experiments}

The measurements are conducted in similar weather and temperature conditions. All experiments are conducted during April in the timespan 11am - 1pm. They are also performed in sunny weather and in a temperature range of $16 \mathrm{C}-20 \mathrm{C}$.

In all of the experiments the end-node device transmits a package with a 2 byte payload every 10 second with a transmitting power of $14 \mathrm{dBm}$. The spreading factor can vary from SF7 to SF12. The SF value is chosen automatically based on the time it takes for the gateway to acknowledge the packet. For all experiments, except the gateway elevation, the experiment is done in two forms:

- with the antennas aligned that means both antennas are vertical,

- when the antennas are misaligned when one antenna is horizontal and pointing towards the other that is positioned vertically.

Open space. The equipment set up on a location where we have at least one kilometer with a free line of sight. The gateway is stationary and end-node device we move far and far away from the gateway, and on each 300 meters we do a measurement. As soon as we lose a single data-packages in the transmissions we decreased the step to 100 meters. We then continue until either 0 packages are received or we run out of space that still maintain free line of sight.

Dense forest. In this test we find a location where the terrain was relatively flat and with a dense vegetation. We then do the measurements starting at 300 meters. The distance is increased by 100 meters each step since we can expect the signal to drop more drastically compared to open space experiment. Gateway is stationary, end device gets moved further away on a straight line. Urban. In this test we do our measurements inside of a city that has houses made of a variation of materials that are located on the same height. We start our measurements at 150 meters and increasing the range by another 150 meters for each measurement while maintaining the same increment of houses for each measurement.

Gateway elevation. For this test we place the gateway and end-node 400 meters apart from one another. We then elevate the gateway in steps of 10 meters starting at 0 while moving the gateway to maintain a $400 \mathrm{~m}$ distance between the two making measurements each step. The device is partly stationary while the device is continuously getting elevated.

\section{Results}

For the experiment in open space results are presented on Fig. 3. At 0m distance we see a clear difference in RSSI between the aligned and misaligned antennas but no difference when it comes to SNR. As the distance increases this difference gets smaller for RSSI and larger for SNR. After 900m the first package loss is observed for the misaligned antenna. Aligned antenna however led to 0 packets lost even at our maximum distance of $1100 \mathrm{~m}$ where we ran out of space.

These results show that even at a distance of $+1100 \mathrm{~m}$ we still have a strong signal that only fades at a tiny rate if at all which could potentially last for 
several more kilometers. In this scenario the LoRa technology is very suitable and the limitation is primarily given by the specification of LoRa itself namely the trade-off between range and bandwidth.
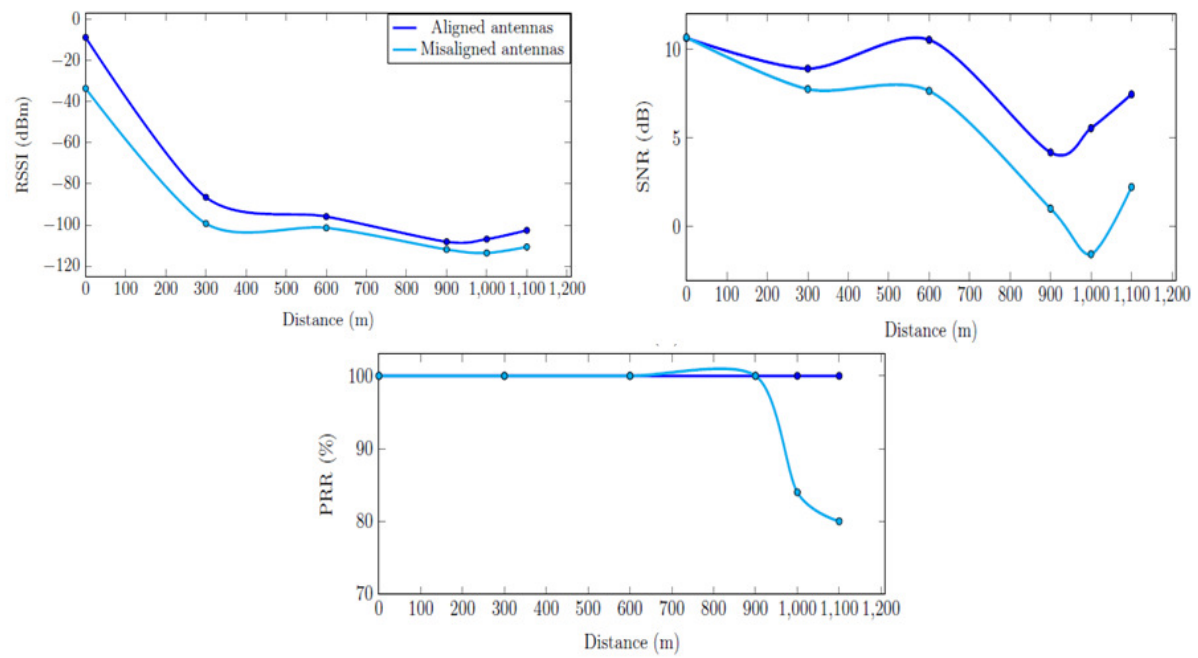

Fig. 3. Open space experiment results.

In the dense forest the SNR starts to decrease after only 300 meters and reaches negative numbers on misaligned antennas at $400 \mathrm{~m}$ and for the aligned at 500m Fig. 4. This would indicate that this environment has a big impact on SNR and by proxy on RSSI as well. SNR however doesnt follow a similar structure as a packet can seemingly be lost at an average of $-5 \mathrm{~dB}$ while a packet with $-13 \mathrm{~dB}$ can be read without problem. The dense forest environment did not affect the connectivity of LoRa.

The urban city experiment shows more drastic drop in both RSSI and SNR (Fig. 5) than in the previous experiments and it can be observed that the values become lower at $150 \mathrm{~m}$ than that of the previous experiments at $300 \mathrm{~m}$. For the next distance measurement step to $300 \mathrm{~m}$ an extreme drop in signal quality can be observed where the PRR for having the antennas aligned gets as low as $32 \%$ while not a single packet can be read while having them misaligned.

While packets seemingly get lost due to poor signal quality the packets that actually get through maintain a mean SNR value of $-7.1 \mathrm{~dB}$ while for the experiment in the forest the value could reach as low as $-13.6 \mathrm{~dB}$ without the packet being lost. Gateway elevation improves the RSSI and gives a value of $-75 \mathrm{dBm}$ at $20 \mathrm{~m}$ height, after this RSSI value is basically unchanged up to 40 meters, the SNR value however continues to rise although at a decaying rate.

Height has as we expected a great impact on the performance to the point where free line of sight and the fresnel zone is clear of obstacles is achieved from 

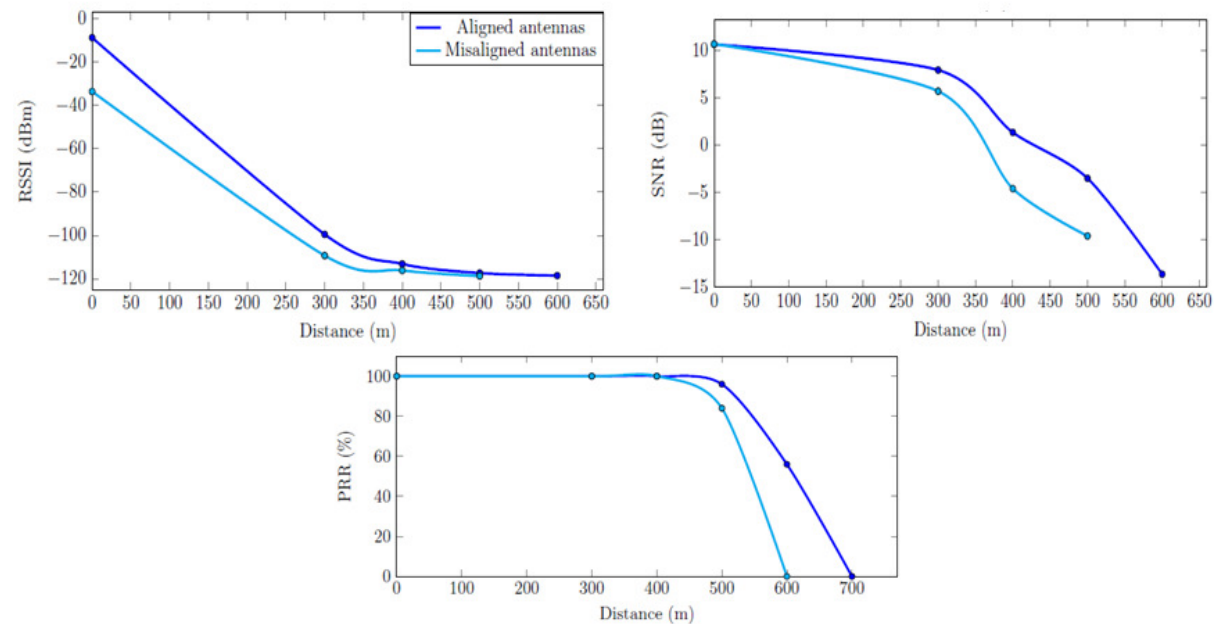

Fig. 4. Dense forest experiment results.

that point on any further elevation might not be considered useful. Applying this elevation to the forest and city that were previously tested would most likely improve the performance greatly and allow for use cases that would otherwise be considered impossible, i.e. large forest agriculture and city based solution that span $+1 \mathrm{~km}$.
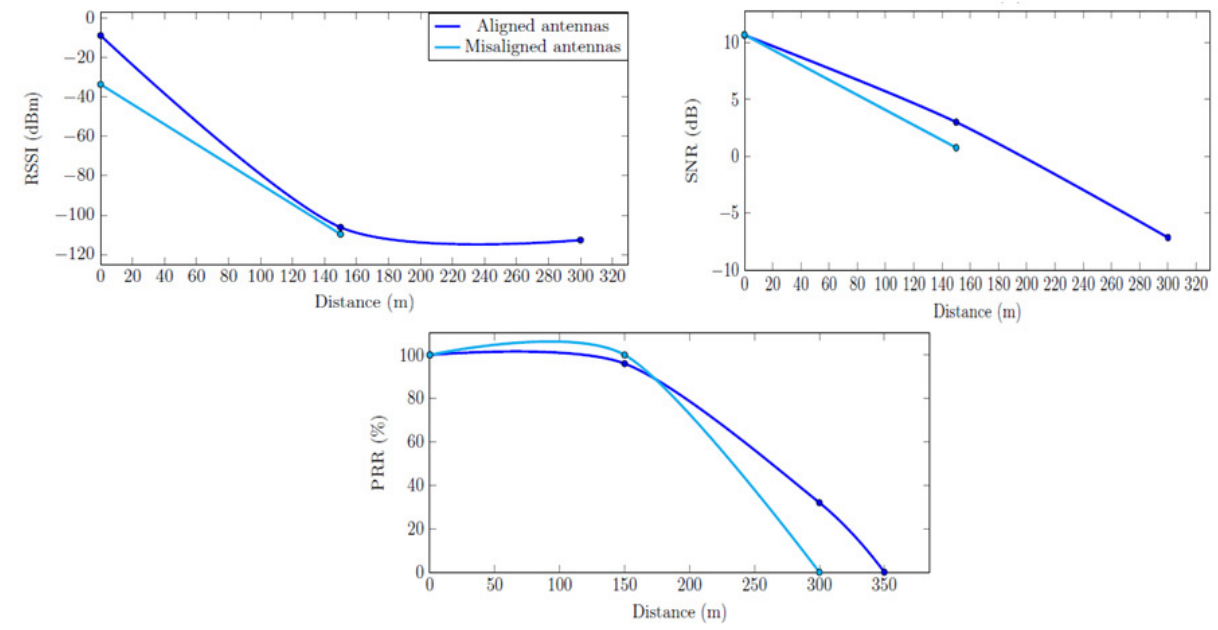

Fig. 5. Urban experiment results. 

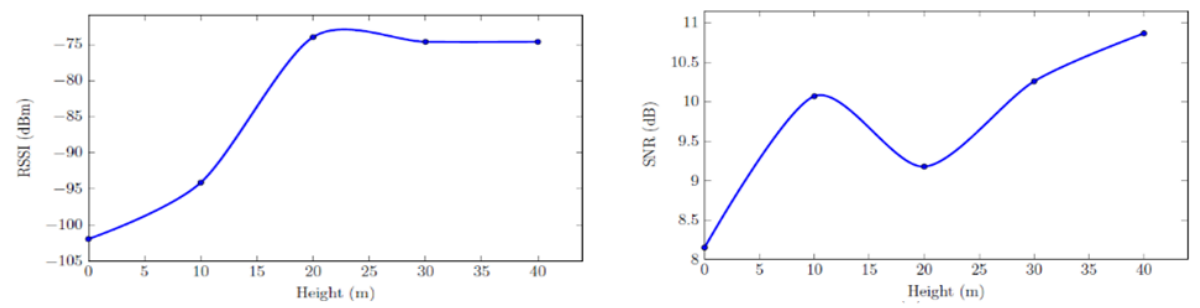

Fig. 6. Gateway elevation experiment results.

\section{Conclusions and future work}

The goal of this paper was to do a performance evaluation of a LoRa network and analyse how the limitation set by the performance affect various use cases.

In the open space the signal keeps a consistently good quality. In the forest and city tests the quality is significantly worse to the point that with our setup in a city environment reaching distances above $350 \mathrm{~m}$ is impossible due to the high amount of noise and quantity of dense materials. This problem can be circumvented by elevating the gateway and/or device to the point that free line of sight or close to it is achieved.

This means that large scale implementations deploying a gateway on a radio tower to prevent obstructions would have no problems reaching distances of $+15 \mathrm{~km}$. For implementations on a lower scale, problems can arise as barriers in the form of houses, terrain and/or vegetation quickly add up and in most cases limits the implementations range to a house or neighborhood. In some cases, elevating the gateway could be impossible then the solution is to use several gateways just like a normal telecommunication solution where a device can connect to any radio tower. Another factor is if the device is mobile as having misaligned antennas leaves a large mark on the performance and there can more than a $50 \%$ difference in PRR.

Regarding packet loss it appears that exceeding the threshold of $-120 \mathrm{dBm}$ causes the packet to become corrupted, i.e. unreadable or lost completely. SNR, however, seems to have less of an effect as a packet is seemingly as readable at $-14 \mathrm{~dB}$ as it is at a positive value. This means that for most implementations it is very important to have packet loss in regard when planning placement as the RSSI can fluctuate and as in most cases there are no retransmissions in LoRa if the device is at the edge of the communication range the packet can be lost forever. This can be very painful for a lot of use cases as the duty cycles are very limited and the next transmission can be hours away.

For future investigation into this subject more case studies are needed, since this one is limited in long term tests and conducted under similar weather conditions for all of the test cases. Furthermore, an extensive comparison to theoretical and simulated results is needed to provide information about how the real-world performance compares to a simulated one as there is always external variables affecting that is not otherwise accounted for. 


\section{References}

1. Sorrell, S.: The Internet of Things: Consumer, Industrial \& Public Services 20162021. Juniper (2016)

2. IoT Market Forecasts, https://www.postscapes.com/internet-of-things-marketsize/. Last accessed 29 May 2018

3. Ericsson Mobility Report. Ericsson (2016)

4. A Comprehensive Look at Low Power, Wide Area Networks. Link Labs, Inc. (2016)

5. Lethaby, N.: Wireless connectivity for the Internet of Things : One size does not fit all. Texas Instrumentals (2017)

6. Adelantado, F. et al.: Understanding the Limits of LoRaWAN. IEEE Communications Magazine 55(9), 34-40 (2017)

7. LoRaWAN 1.1 Regional Parameters. LoRa Alliance, Inc. (2017)

8. LoRaWAN 1.1 Specification. LoRa Alliance, Inc. (2017)

9. Mekki, K. et al.: A comparative study of LPWAN technologies for large-scale IoT deployment. ICT Express (2018).https://doi.org/10.1016/j.icte.2017.12.005

10. Centenaro, M. et al.: Long-range communications in unlicensed bands: the rising stars in the IoT and smart city scenarios. IEEE Wireless Communications 23(5), 60-67 (2016)

11. Cattani, M., Boano, C., Rmer, K.: Experimental Evaluation of the Reliability of LoRa Long-Range Low-Power Wireless Communication. Journal of Sensor and Actuator Networks 6(2), 7 (2017)

12. Wennerstrm, H. et al. : A long-term study of correlations between meteorological conditions and 802.15.4 link performance. In: IEEE International Conference on Sensing, Communications and Networking, pp.221-229. IEEE, New Orleans, LA, USA (2013)

13. Ferre, G.: Collision and packet loss analysis in a LoRaWAN network. In: 25th European Signal Processing Conference, pp. 2586-2590. IEEE, Kos, Greece (2017)

14. European Commission ERC Recommendation 70-03, https://www.efis.dk/sitecontent.jsp?sitecontent=srd_regulations. Last accessed 29 May 2018

15. Petjjrvi, J. et al.: Performance of a low-power wide-area network based on LoRa technology: Doppler robustness, scalability, and coverage. International Journal of Distributed Sensor Networks 13(3) (2017)

16. Thiemo,V., Utz, R., Bor, M., Juan, A.: Mitigating Inter-network Interference in LoRa Networks. In: Proceedings of the 2017 International Conference on Embedded Wireless Systems and Networks, pp. 323-328. ACM, Uppsala, Sweden (2016)

17. Alos, A. et al.: A Study of LoRa: Long Range \& Low Power Networks for the Internet of Things. Sensors (Basel). 16(9), 1466 (2016)

18. ST. I-CUBE-LRWAN. http://www.st.com/en/embedded-software/icube- lrwan.html.. Last accessed 29 May 2018

19. LoRa Gateway Project. https://github.com/Lora-net/lora_gateway. Last accessed 29 May 2018 